\title{
The Muon $g-2$ Experiment at Fermilab: Commissioning and First Physics Data Taking
}

\author{
James Stapleton*广 \\ Fermi National Accelerator Laboratory \\ E-mail: jstapletefnal.gov
}

Extremely precise measurements of the muon magnetic dipole moment two decades ago revealed a tantalizing hint of an anomalous excess at the sub-ppm level over Standard Model predictions. This $\sim 3.5$ sigma discrepancy has been widely cited due to the anomaly's potential for constraining many natural extensions to the Standard Model. A new experiment at the Fermi National Accelerator Laboratory will be able to refine this measurement by a factor of four over the next few years by measuring the spin precession of $3.1 \mathrm{GeV}$ muons trapped in a precisely tuned magnetic field. This talk offers an update on the early stages of the experiment and our expectations for accumulating the required statistics.

XIV International Conference on Heavy Quarks and Leptons (HQL2018)

May 27- June 1, 2018

Yamagata Terrsa, Yamagata, Japan

\footnotetext{
*Speaker.

${ }^{\dagger}$ On behalf of the Fermilab Muon $g-2$ Collaboration
} 


\section{Introduction}

The measurement of magnetic dipole moments has a long history of challenging the most stringent applications of theories of idealized point particles: the $\sim 0.2 \%$ deviation of the electron's dipole moment was a critically precise test of quantum electrodynamics, while its mere existence in the neutron offered indirect evidence of quark structure as early as the 1930s.

In 2006 the E821 experiment at Brookhaven National Laboratory published a final report of a $\gtrsim 3 \sigma$ excess in the magnetic dipole moment of the muon[1]. This discrepancy generated a great deal of interest because it challenged our understanding of precision effects such as highorder QED loops and hadronic vacuum polarization. More than a decade of refinement in these theoretical computations has pushed the original measurement to $3.7 \sigma$ excess over the Standard Model predictions available to date[2][3].

The 'anomaly' $a_{\mu}$ is defined as the small deviation of the moment's $g$-factor beyond the value of 2 for a classical point-like particle:

$$
g_{\mu}=2\left(1+a_{\mu}\right)
$$

The anomaly would be zero without contributions of $\simeq 10^{-3}$ from dipole self-interaction and higher-order QED loop diagrams, $\simeq 10^{-7}$ from interactions with the hadronic vacuum, and $\simeq 10^{-9}$ from weak interactions. The measured excess amounts to roughly $3 \cdot 10^{-9}$ in the value of $a_{\mu}$, potentially as strong as the interactions which probe the weak sector at high masses. The 540 partper-billion measurement is already one of the most precise of any fundamental particle property and, if the excess is confirmed, it could represent muon-vacuum interactions with anything from MSSM to dark matter.

This groundbreaking measurement was the culmination of decades of work by a small, determined group of experimentalists, an effort stretching back to the early days of CERN. Exploiting the realization by Cassels et al.[4] in 1957 that the spin precession of dynamically-stored muons is more sensitive to $a_{\mu}$ than muons at rest, the storage ring experiment used muons with carefullytuned momenta: a gamma factor $\gamma \sim 29$ extends the lifetime of subject muons to $\sim 64 \mu$ s while the corresponding 'magic momentum' $p \sim 3.1 \mathrm{GeV}$ exactly cancels the spin precession contributions from the electrostatic quadrupoles used to maintain vertical focusing during storage. Injection into a precisely-controlled 1.45 Tesla dipole magnetic field traps the beam in the circular storage ring for $\gtrsim 170$ periods of the $\omega_{a}$ oscillation, defined as the spin precession frequency relative to the cyclotron frequency of the muon momentum vector:

$$
\begin{aligned}
\vec{\omega}_{a} & \equiv \vec{\omega}_{\text {spin }}-\vec{\omega}_{\text {cyclotron }} \\
& =-\frac{q}{m}\left[a_{\mu} \vec{B}-\left(a_{\mu}-\frac{1}{\gamma^{2}-1}\right) \frac{\vec{\beta} \times \vec{E}}{c}\right]
\end{aligned}
$$

or simply $-a_{\mu} q \vec{B} / m$ for $\gamma_{\text {magic }}^{2} \equiv 1+1 / a_{\mu}$.

The physical mechanism of the measurement itself is exceedingly simple: correlations between muon spin and the momentum of its $e^{ \pm}$decay products imprints an oscillating energy asymmetry upon their outgoing direction in the lab frame. We effectively 'image' the muon spin precession through directional measurement of the time and energy spectrum of their decay products. 
This induces a distinct oscillation in the time histogram of the highest-energy positrons captured. An example is figure $1 b$.

\section{The New Experiment at Fermilab}

The rigorous science program of the Alternating Gradient Synchrotron at Brookhaven limited the run time of the E821 experiment, but within a few years of the final report there emerged a significant effort to continue the experiment at Fermilab. With the decommissioning of the Tevatron and subsequent refitting aimed at high intensity production of medium-energy pions, Fermilab offered a unique opportunity to revive the storage ring experiment. We have leveraged the existing facilities and expertise in order to produce one of the world's highest intensity beams of polarized muons. With a comparable intensity, higher duty cycle, and strongly suppressed pion contamination compared to the AGS, the new beam line can deliver in 400 to 500 days of run time enough muons to generate a spin precession dataset roughly 20 times larger than that of E821, while reducing beam injection systematics.

The original E821 ring was transported from Brookhaven to Fermilab due to the prohibitive cost of manufacturing another one. It could not be transported standing on its edge, but with a diameter nearly four traffic lanes wide it could not be carried by road. Eventually it was decided to carry the ring by barge down the Eastern coast of the United States, around the Florida peninsula, into the Gulf, and up the Mississippi River to Illinois, where it was unloaded and transported the last few dozen miles to Fermilab. The entire journey took over a month to complete, and the last part of the journey consisted of several trips executed solely at night because it required a complete shutdown of one side of the freeway outside of Chicago.

The new installation aims to improve upon the original measurement on three fronts. While the larger dataset by itself may push the observed excess to much higher significance, we expect that we can improve magnetic field uniformity and precession measurement systematics by taking advantage of the cleaner muon beam and advancements in detector technologies since the Brookhaven experiment. We expect to achieve precision as good as $140 \mathrm{ppb}$ on the value of $a_{\mu}$.

\subsection{Muon Beamline and Injection into the Storage Ring}

The muon beamline now operating at Fermilab utilizes the LINAC, booster ring, recycler ring, and a portion of the Tevatron's main injector to produce an $8 \mathrm{GeV}$ proton beam on the existing antiproton source's Inconel target for pion production. This delivers more than $10^{11}$ protons per cycle at an average rate of 12 cycles per second. This produces pions in large amounts which are steered through the old antiproton line where the beam is conditioned and separated from residual protons, then directed through the debunching ring where several laps provide enough time for decay to muons and separation of these from remaining pions.

Delivered to the 'Muon Campus (MC) 1' experiment hall and handled by the last few focusing quadrupoles, the beam is injected into the ring through a small hole in the magnetic yoke steel and guided into the storage region by the inflector, a special superconducting tube used to shield the approaching muons from fringe field gradients of the magnetic dipole field. The momentum acceptance of the storage ring is constrained to roughly $0.2 \%$ variation about the magic momentum, which limits muon storage to $10 \mathrm{k}$ to $20 \mathrm{k}$ per 'fill'. 
The storage region is defined as a $9 \mathrm{~cm}$ diameter tunnel (torus) housed in aluminum vacuum chambers to prevent premature energy loss through interactions with atmosphere. The center of this tunnel is at a radius of $7.112 \mathrm{~m}$ from the center of the ring, where the muons' synchrotron radius corresponds to the 'magic momentum'. The muon beam is focused vertically by the electrostatic quadrupoles, and horizontally by the combined effect of these and the magnet's dipole field.

Muons are initially injected onto a circular orbit displaced $77 \mathrm{~mm}$ from the exact center of the ring. Fast electromagnetic kickers are used in the first turn to deflect the muons onto a properlycentered orbit, and kick amplitude provides a handle to tune the initial phase for horizontal betatron motion. The electrostatic quadrupoles are constructed so that in some sections, for a short time after injection, the beam can be intentionally displaced toward the outer or upper edge of the storage region against solid metal collimators. This 'scrapes' off the muons in the least desirable portion of the accepted kinematic phase space, ensuring that most of the remaining muons are well-centered within the storage region.

\subsection{Muon Storage Ring and Dipole Field}

The storage ring itself consists of three coils of Niobium-Titanium superconducting cable encased in a copper matrix with a design based on the TOPAZ solenoid used for the TRISTAN accelerator in Nippon[5]. With $\sim 6$ tons of cold mass carrying nearly 5 kiloAmperes (and $\sim 680$ tons of iron total) the ring stores 6 megajoules of energy when fully powered. The coils are installed on the inner radius of a metal frame including 'yoke' steel to extend and stabilize the magnetic field, and adjustable steel pole pieces extend from the yoke toward the center plane to diminish field irregularity in the muon storage region. Steel wedge-shaped shims installed between the yoke and pole pieces counteract the intrinsic quadrupole moment induced by the yoke's shape. The shims' positions can be radially adjusted, affecting only the dipole component of the field. The small radial component of the magnetic field is tuned using copper coils carrying a few hundred $\mathrm{mA}$ installed on the inner wall of the pole pieces. Inserted between the pole pieces are aluminum vacuum chambers to prevent muon energy loss through interactions with atmosphere. The vacuum chambers and yoke steel are assembled in 12 sections each covering $30^{\circ}$ of azimuth, each housing three $10^{\circ}$ sections of upper and lower pole pieces.

In E821 the dipole field was regulated to a uniformity of $1 \mathrm{ppm}$ averaged over azimuth, and the new installation at Fermilab achieved a factor $3 \times$ better in 2016. This is accomplished using NMR probes to measure the spin precession frequency of protons as a proxy for the magnitude of the magnetic field. A series of 378 probes are installed at fixed locations next to the pole pieces, and they are cross-calibrated by probes installed on a movable 'trolley' which travels the full azimuth of the ring to measure the field's cross-section in the storage region. Trolley runs are performed regularly during scheduled or unscheduled beam down times.

\subsection{Major Detector Systems}

The major detector systems are a series of positron calorimeters installed around the azimuth which capture positrons as they spiral toward the center of the ring, and straw trackers positioned in front of two of the calorimeters. Segmented calorimeters intercept decay positrons in a $6 \times 9$ array of small lead-fluoride crystals. The positron generates $e^{ \pm}$showers whose Čerenkov photons 


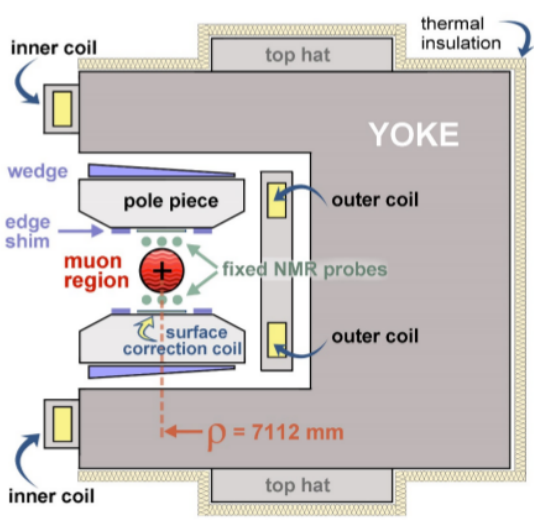

(a) ring cross-section

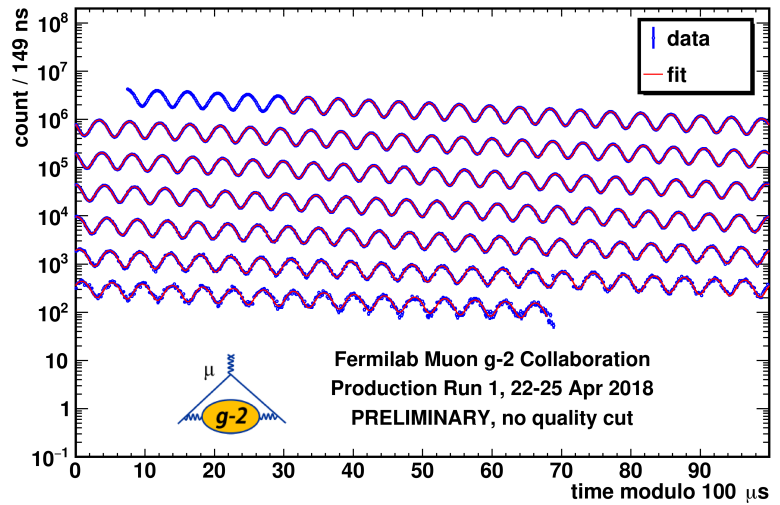

(b) Latest Wiggle Plot

Figure 1: a) Yoke steel, superconducting coils, and the elements used to shape the magnetic field near the muon storage region. The center of the ring is to the left. b) This figure was accumulated from 60 hours of data from 22 - 25 Apr 2018 and has approximately 0.95 billion positrons. The number of wiggles is similar to the one achieved by BNL in 1999.

are measured by silicon photomultipliers at the rear at a depth of $14 \mathrm{~cm}$. This is a significant improvement over the E821 positron calorimeters, which were not truly segmented and could not reconstruct single positrons with a position and energy to the same degree. Sensitive to positrons between tens of $\mathrm{MeV}$ to a few $\mathrm{GeV}$, their energy resolution of a few percent is more than adequate for the purpose of measuring the energy-dependent oscillating asymmetry in positron production which encodes the muon spin precession. The acceptance of the calorimeters to all decay positrons is roughly one-third due to the calorimeters' height (just over $15 \mathrm{~cm}$ ) and the dipole field's tendency to bend decay positron trajectories inward[6][7].

The gain stability of the silicon photomultipliers is monitored closely, greatly improving the benefit of positron hit reconstruction. The 'laser hut', a separate booth in the experiment hall outside the storage ring, houses laser production and control equipment which serves every crystal of every calorimeter plus a few ancillary detectors. Six laser heads are fired roughly simultaneously, and optical fibers carry their signal to the inside of the ring where each source is split and routed into four calorimeters. Inside the calorimeter installation, the signal is split again into (at least) 54 separate optical fibers by a diffuser, routed to a 'faceplate' delivers optical light into each crystal[8].

Two straw trackers each consist of 8 double layers of aluminized mylar drift tubes which measure ionization in argon-ethane gas. They are used primarily to track positrons back to muon decay positions in order to deduce the spatial distribution of muons in the storage region. This represents another significant improvement over E821 as we can convolve this distribution with the magnetic field measured by trolley probes, which reduces the overall systematics on muon spin precession measurement[9]. 


\subsection{The Measurement and its Uncertainties}

The final determination of the dipole moment anomaly composes it as a product of factors:

$$
a_{\mu}=\frac{g_{e}}{2}\left(\frac{\omega_{a}}{\omega_{p}}\right)\left(\frac{m_{\mu}}{m_{e}}\right)\left(\frac{\mu_{p}}{\mu_{e}}\right) .
$$

(See the Technical Design Report[10] for a derivation.) The factors external to this experiment are known to high precision, with $\delta g_{e}=0.28 \mathrm{ppt}, \delta\left(m_{\mu} / m_{e}\right)=25 \mathrm{ppt}$, and $\delta\left(\mu_{p} / \mu_{e}\right)=8 \mathrm{ppb}$. The contributions to uncertainty on $\omega_{a} / \omega_{p}$ are well understood due to the maturity of the experiment, and the new installation at Fermilab includes a concrete, achievable roadmap to reducing the uncertainty from each source. The target for $\omega_{a}$ is $100 \mathrm{ppb}$ (stat.) $+70 \mathrm{ppb}$ (syst.), a significant improvement over E821. The reduction in statistical uncertainty is of course due to the factor $\sim 20 \times$ more data expected in the Fermilab operation, while the upgraded detector and beam control elements allow reduction of all of the major systematic uncertainties which affected the E821 result.

One of the largest systematics for the previous experiment's precession measurement was gain variations in the PMTs reading out the calorimeters; the new laser-based gain calibration system and the change to silicon photomultipliers is expected to reduce this contribution from $120 \mathrm{ppb}$ to as low as $20 \mathrm{ppb}$. The $6 \times 9$ segmented calorimeter design greatly improves our ability to resolve coincident positron hits and hits from muons (which occasionally lose energy and leak out of the storage region) reducing by a factor of 2 and 4, respectively, the uncertainty contributions from each effect.

The remaining $\omega_{a}$ systematics are mostly the effect of deviations from the ideal path in the storage region. Muons not centered in the storage region undergo horizontal and vertical betatron motion, causing the muon bunch to orbit a fixed point in phase space composed of displacement distance from the center and lateral displacement of the momentum vector from ideal circular motion. While the segmented calorimeters can detect this beam motion much better than the monolithic design, the real improvement here is due to the straw trackers: their ability to track positrons back to the storage region gives us a direct picture of the muon beam's movement and is expected to reduce the uncertainty contribution from horizontal and vertical motion by a factor of two each.

We quantify the magnetic field strength according to $\omega_{p}$, the spin precession rate for protons, using NMR probes. The field strength directly affects the relationship between spin precession rate and the dipole moment, and $\omega_{p}$ therefore has a commensurate contribution to the uncertainty on $a_{\mu}$. The Fermilab experiment targets an $\omega_{p}$ uncertainty of $70 \mathrm{ppm}$, reduced from the Brookhaven iteration's $170 \mathrm{ppm}$. Gains come from improved magnet shimming and temperature stability as well as from a variety of monitoring improvements, including more frequent calibrations, a larger number of fixed probes, and upgraded electronics. The trolley design was significantly updated according to experience from the Brookhaven experiment, boasting movable probes, better position control and monitoring, more probes at larger radii in the storage region, an improved power supply, and resilience to temperature effects.

\section{Status and Outlook}

The muon beamline and storage ring were first commissioned in a run over several weeks in 
Summer 2017. Run time typically consisted of alternating shifts of muon beamline tuning and ring data-taking. The outcome was a detailed understanding of the performance of a number of subsystems from beamline efficiency to offline data handling capacity.

The first true physics run, from January to June 2018, produced a dataset roughly $2 \times$ the size of that from E821, and has been followed by an aggressive campaign to identify efficient data quality cuts, quantify systematic uncertainties, and prepare for the publication next year of a dataset which could yield exciting results. In May 2018 we entered a planned shutdown period and began a rigorous program of upgrades, maintenance, and repairs which are expected to boost efficiency in a number of areas. After starting up again this fall we charge ahead into the task of collecting a dataset which will simultaneously overshadow that of E821 while shining a light on its tantalizing but persistent hint of new physics.

This work was supported in part by U.S. Department of Energy and Fermilab (under contract No. DE-AC02-07CH11359) among other institutions. See http://muon-g-2.fnal.gov/ for more information.

\section{References}

[1] Bennett GW et al. (Muon $g$ - 2 Collaboration), Phys. Rev. D 73, 072003 (2006)

[2] Keshavarzi A, Nomura D, Teubner T, The Hadronic Vacuum Polarisation Contributions to the Muon $g-2$ (arXiv:1802.06229)

[3] Blum et al. "Calculation of the Hadronic Vacuum Polarization Contribution to the Muon Anomalous Magnetic Moment", Phys. Rev. Lett. 121, 022003 (2018)

[4] Cassels J et al. 1957 Proc. Phys. Soc. A 70543.

[5] Yamamoto A, Inoue H, Hirabayashi H, J. Phys. 45 (1984) C1-337.

[6] Fienberg A et al. "Studies of an array of $\mathrm{PbF}_{2}$ Čerenkov crystals with large-area SiPM readout", Nucl. Instrum. Meth. A783 (2015) 12-21, arXiv:1412.5525

[7] Kaspar $\mathrm{J}$ et al. "Design and performance of SiPM-based readout of $\mathrm{PbF}_{2}$ crystals for high-rate, precision timing applications", JINST 12 (2017) no.01, P010009

[8] Karuza M et al. (Muon $g-2$ Collaboration), "The Fermilab Muon $g-2$ experiment: laser calibration system," JINST 12 (2017) no.08, C08019.

[9] Mott J (Muon $g-2$ Collaboration), "The readout system for the Fermilab Muon $g-2$ straw tracking detectors", PoS ICHEP2016 (2017) 1163

[10] Grange J et al. (Muon $g-2$ Collaboration), "Muon $(g-2)$ Technical Design Report" (arXiv:1501.06858, Fermilab Report No. FERMILAB-FN-0992-E, FERMILAB-DESIGN-2014-02) 